\title{
Spectral characteristics of water megamaser galaxies
}

\section{ESO 103-G035, TXS 2226-184, and IC 1481^}

\author{
N. Bennert ${ }^{1}$, H. Schulz ${ }^{1,2, \star \star}$, and C. Henkel ${ }^{3}$ \\ 1 Astronomisches Institut Ruhr-Universität Bochum, Universitätsstraße 150, 44780 Bochum, Germany \\ 2 Universidad Católica del Norte, Avenida Angamos 0610, Antofagasta, Chile \\ 3 Max-Planck-Institut für Radioastronomie, Auf dem Hügel 69, 53121 Bonn, Germany \\ e-mail: chenkel@mpifr-bonn.mpg.de
}

Received 13 October 2003 / Accepted 25 February 2004

\begin{abstract}
Long-slit optical emission-line spectra of the $\mathrm{H}_{2} \mathrm{O}$ megamaser galaxies ESO 103-G035, TXS 2226-184, and IC 1481 are evaluated in order to look for characteristics typical for water-megamaser galaxies. We present rotation curves, line ratios, electron densities, temperatures, and $\mathrm{H} \beta$ luminosities. The successful line-profile decompositions rest on $d$-Lorentzians with an additional parameter $d$ to adjust the wings, rather than Gaussians or Lorentzians as basic functions. No significant velocity gradient is found along the major axis in the innermost $2 \mathrm{kpc}$ of TXS 2226-184. IC 1481 reveals a spectrum suggestive of a vigorous starburst in the central kiloparsec $10^{8}$ years ago. None of the three galaxies shows any hints for outflows nor special features which could give clues to the presence of $\mathrm{H}_{2} \mathrm{O}$ megamaser emission. The galaxies are of normal Seyfert- 2 (ESO 103-G035) or LINER (TXS 2226-184, IC 1481) type.
\end{abstract}

Key words. galaxies: active - galaxies: nuclei - galaxies: individual: ESO 103-G035, TXS 2226-184, IC 1481

\section{Introduction}

Extragalactic $\mathrm{H}_{2} \mathrm{O}$ megamaser sources are orders of magnitude more powerful than strong $\lambda=1.3 \mathrm{~cm}$ Galactic masers of the same type and have been discovered in about three dozen galactic nuclei, almost all of them of Seyfert-2 or LINER (lowionization nuclear emission-line region) type (e.g. Braatz et al. 1996; Greenhill et al. 2003).

According to the so-called unified model (Antonucci \& Miller 1985; Antonucci 1993), Seyfert-2 objects contain a nuclear molecular torus seen edge-on that surrounds an X-ray luminous, winds and jets producing active galactic nucleus (AGN). It is plausible that this geometry is favorable for megamaser activity, because the necessary large column densities of warm $(T \geq 400 \mathrm{~K})$ and dense $\left(n\left(\mathrm{H}_{2}\right) \geq 10^{7} \mathrm{~cm}^{-3}\right)$ molecular gas enriched with $\mathrm{H}_{2} \mathrm{O}$ molecules are likely to be supplied. Interaction of molecular gas with a radio jet may also cause conditions favorable for the occurrence of $\mathrm{H}_{2} \mathrm{O}$ masers (Peck et al. 2003).

This paper is the second one in a series analyzing the spectral properties of water-vapor megamaser galaxies at optical wavelengths. In the first one (Schulz \& Henkel 2003),

Send offprint requests to: $\mathrm{N}$. Bennert,

e-mail: nbennert@astro.rub.de

* Based on observations collected at the European Southern Observatory, Chile.

$\star \star$ Deceased. emission-line profiles of IC 2560, NGC 1386, NGC 1052, and Mrk 1210 were evaluated. Galactic rotation and outflow of narrow-line gas are common features of this sample of water-megamaser galaxies. All decomposed line-systems exhibit AGN typical line ratios. For NGC 1052 and Mrk 1210, maser emission triggered by the optically detected outflow components cannot be ruled out.

In this work, we continue to explore the structure of galactic nuclei containing $\mathrm{H}_{2} \mathrm{O}$ megamaser sources (ESO 103-G035, TXS 2226-184, IC 1481) in order to look for distinguishing characteristics and to obtain clues to the nuclear geometry.

\subsection{Individual objects}

Marshall et al. (1979) and Piccinotti et al. (1982) classified ESO 103-G035 (IRAS 18333-6528) as a Seyfert-1.9 galaxy whereas Morris \& Ward (1988) did not find any hints of broad line wings. Observations with EXOSAT (European Space Agency's X-ray Observatory) revealed variable, strong absorption of soft X-rays (Warwick et al. 1988). Heisler \& Vader (1994) find ESO 103-G035 among the warmest far-infrared emitters in their sample of IRAS (Infrared Astronomical Satellite) galaxies with spectral energy distributions peaking near 60 microns. It unveals a high excitation emission-line spectrum superposed on a red stellar continuum with absorption lines. Braatz et al. (1996) discovered $\mathrm{H}_{2} \mathrm{O}$ megamaser emission in this galaxy. 
So far, the most luminous known $\mathrm{H}_{2} \mathrm{O}$ maser is found in the galaxy TXS 2226-184 (IRAS F22265-1826; Koekemoer et al. 1995). Hubble Space Telescope (HST) and Very Large Array observations from Falcke et al. (2000) suggest that it is a galaxy of type later than S0 with an inclination of $\sim 70^{\circ}$. Their $\mathrm{H} \alpha+[\mathrm{N} \mathrm{II}]$ map exhibits a gaseous, jetlike structure perpendicular to the galaxy's major axis and its nuclear dust lane. The 8.4 GHz radio continuum map shows emission that is elongated in the same direction as the $\mathrm{H} \alpha+[\mathrm{N}$ II] emission. Falcke et al. (2000) concluded that the nuclear accretion disk, the obscuring torus, and the large-scale molecular gas layer are roughly coplanar. Taylor et al. (2002) found H I in absorption towards TXS 2226-184, consisting of two components with widths of $125 \mathrm{~km} \mathrm{~s}^{-1}$ and $420 \mathrm{~km} \mathrm{~s}^{-1}$, respectively. They suggest that the $\mathrm{HI}$ absorption is produced in the central parsecs of the galaxy, on a scale similar to that which gives rise to the water maser emission.

Little is known about IC 1481 (IRAS 23168+0537) which was classified as LINER by Huchra et al. (1982). Braatz et al. (1996) discovered its water megamaser emission. Due to its amorphous appearance (e.g. Falcke et al. 2001) its inclination is hard to determine: De Vaucouleurs et al. (1991) (RC3) list an inclination of $i \sim 70^{\circ}$ whereas $i \sim 30^{\circ}$ is given in Braatz et al. (1997). van den Bergh et al. (2002) classify IC 1481 in their catalogue of host galaxies of supernovae as a peculiar $\mathrm{Sb}$ galaxy.

\section{Observations and data reduction}

The spectra described here were obtained by H. Domgörgen in May 1996 using the Boller \& Chivens spectrograph attached to the Cassegrain focus of the European Southern Observatory (ESO) $1.52 \mathrm{~m}$ telescope. Observations were made in two spectral ranges (3400-5400 $\AA$ and 5400-7400 $\AA$ ) through the nucleus along the major axis of each galaxy with exposure times ranging between $1800 \mathrm{~s}$ and $3600 \mathrm{~s}$. A $\log$ of the observations is given in Table 1. The detector used was La Silla CCD No. 39 (Loral Lesser) with $15 \mu \mathrm{m}$ wide square pixels. The spatial resolution element is 0 .' $^{\prime} 68 \mathrm{pix}^{-1}$. Seeing and telescope properties limited the spatial resolution to the range 1 .' $2-2$ '.5 which was determined by the width of spectra of standard stars on the focal exposures. The $2^{\prime \prime}$ wide slit projects to a spectral resolution of $\sim 2.7 \AA\left(\sim 130 \mathrm{~km} \mathrm{~s}^{-1}\right)$ as is confirmed by the full width at halfmaximum $(F W H M)$ of comparison lines and the [O I] $\lambda 6300$ night-sky line.

Standard reduction including bias subtraction and flat-field correction was performed using the ESO MIDAS ${ }^{1}$ software (version Nov. 98). Night-sky spectra "above" and "below" any notable galaxy emission were interpolated in the region of the galactic spectrum and subtracted in each case. Excellent flux interpolation was achieved by rebinning the spectra to a scale of $0.97 \AA$ per pixel during wavelength calibration. The curve of Tüg (1977) was used to correct for atmospheric extinction. The spectra were flux calibrated using the standard stars LTT 7987 or CD $-32^{\circ} 9927$.

\footnotetext{
${ }^{1}$ Munich Image Data Analysis System, trade-mark of the ESO.
}

From the frames cleaned in this way, single rows were extracted (each row corresponding to 0.'68 and 2" along and perpendicular to the slit direction, respectively). Along the "spatial axis" of the CCD, we identified the "photometric center" (that we choose as the "zero" on the spatial scale) with the most luminous row of the CCD ("central row"); this does not have to coincide with the dynamical center. NGC 3115 or NGC 4179 (both classified as S0 galaxies in the NASA/IPAC Extragalactic Database (NED)) were used as templates to subtract the stellar contribution from each single row. These template spectra turned out to be rather similar in our spectral ranges and were usually suitable to fit the absorption lines of the megamaser galaxies. Only for IC 1481, a special treatment was necessary that will be discussed in the next section.

For line ratios presented here, the three central rows were averaged to enhance the $S / N$ without loosing any spatial information. Hence, the line ratios refer to the central region of $2^{\prime \prime} \times 2^{\prime \prime}$. Forbidden-line wavelengths were taken from Bowen (1960). Heliocentric corrections as given in Table 1 were added to the observed velocities. The sample properties are given in Table 2 .

\section{Results}

The dereddened spectra of the innermost regions $\left(2^{\prime \prime} \times 2^{\prime \prime}\right)$ rebinned to rest wavelengths are shown in Fig. 1.

\subsection{Stellar population of the galactic nuclei}

To derive emission-line fluxes, we need to model the stellar population which can contaminate our emission-line spectra due to underlying absorption lines. As a first approximation, we simply subtracted one of the S0-type template spectra scaled to the inter-emission-line parts from all spectra. For two of the three objects, there remains no notable continuum nor absorption-line spectrum. This means that, to our accuracy, the stellar populations in the nuclei of ESO 103-G035 and TXS 2226-184 closely resemble the old stellar populations in NGC 3115 and NGC 4179.

Joguet et al. (2001) classified ESO 103-G035 as "Seyfert 2b" meaning that they observed high order Balmer lines in absorption which are often interpreted to indicate a significant young stellar population. We do not find high order Balmer absorption lines in our spectra. This might be due to our lower $S / N$ spectra: Joguet et al. (2001) used the same telescope and instrument but a UV-blazed grating and a CCD camera with high quantum efficiency below $5000 \AA$. Furthermore, they used twice the integration time with half the spectral resolution. However, having a closer look at their online data, we find that ESO 103-G035 resembles more closely some galaxies which Joguet et al. (2001) classified as "Seyfert 2a" (spectra showing both emission and absorption lines in which the Balmer series is not detected in absorption, e.g. ESO 323+G32) than "Seyfert 2b", thus in agreement with our spectra. In our raw data we see Ca II K $\lambda 3933$, weak $\mathrm{CN} \lambda 4200, G$ band $\lambda 4301$ and $\mathrm{Mg} \mathrm{I} \lambda 5175$ absorption lines. Since all of them can be well fit by NGC 3115, we exclude the possibility of a significant residual starburst contribution. 
Table 1. Summary of the spectroscopic observations.

\begin{tabular}{lcccc}
\hline \hline Object/hel. corr. $^{a}$ & Date (beg.) & PA (slit) & $\lambda$ range $(\AA)$ & Exp. time (s) \\
\hline ESO 103-G035 & 14-May-96 & $44^{\circ}$ & $5400-7400$ & 1800 \\
$+14 \mathrm{~km} \mathrm{~s}^{-1}$ & 17-May-96 & $44^{\circ}$ & $3400-5400$ & 1800 \\
\hline TXS 2226-184 & 14-May-96 & 50.3 & $5400-7400$ & 2700 \\
$+29 \mathrm{~km} \mathrm{~s}^{-1}$ & 17-May-96 & $50^{\circ} 3$ & $3400-5400$ & 2700 \\
\hline IC 1481 & 14-May-96 & $24^{\circ}$ & $5400-7400$ & 3600 \\
$+25 \mathrm{~km} \mathrm{~s}^{-1}$ & 17-May-96 & $24^{\circ}$ & $3400-5400$ & 2400 \\
\hline
\end{tabular}

${ }^{a}$ This heliocentric correction was added to the measured radial velocities.

Table 2. $\mathrm{H}_{2} \mathrm{O}$-megamaser sample properties.

\begin{tabular}{|c|c|c|c|}
\hline & ESO 103-G035 & TXS 2226-184 & IC 1481 \\
\hline & \multicolumn{3}{|c|}{ literature } \\
\hline coordinates $^{a}$ & $18^{\mathrm{h}} 38^{\mathrm{m}} 20^{\mathrm{s}}-65^{\circ} 25^{\prime} 39^{\prime \prime}$ & $22^{\mathrm{h}} 29^{\mathrm{m}} 13^{\mathrm{s}}-18^{\circ} 10^{\prime} 47^{\prime \prime}$ & $23^{\mathrm{h}} 19^{\mathrm{m}} 25^{\mathrm{s}}+05^{\circ} 54^{\prime} 22^{\prime \prime}$ \\
\hline $\operatorname{incl}^{b}\left({ }^{\circ}\right)$ & $67^{\circ}$ & $70^{\circ}$ & $62^{\circ}$ \\
\hline m.a..$^{c}\left({ }^{\circ}\right)$ & $44^{\circ}$ & $51^{\circ}$ & $70^{\circ}\left(30^{\circ d}\right)$ \\
\hline$v_{\mathrm{opt}}^{e}\left(\mathrm{~km} \mathrm{~s}^{-1}\right)$ & $3983 \pm 34$ & - & $6118^{f} \pm 50$ \\
\hline \multirow[t]{2}{*}{ Type $^{g}$} & Sy $1.9 / 2, \mathrm{Sa} 0$ & $?, \mathrm{~S} 0 ?$ & LINER, $\mathrm{Sb} \operatorname{pec}^{f}$ \\
\hline & \multicolumn{3}{|c|}{ this work } \\
\hline$v_{\text {hel }}^{h}\left(\mathrm{~km} \mathrm{~s}^{-1}\right)$ & $4040 \pm 20$ & $7520 \pm 30$ & $6120 \pm 30$ \\
\hline dist. $^{i}(\mathrm{Mpc})$ & 61.9 & 110.6 & 88.5 \\
\hline lin. sc. $\left(p c /{ }^{\prime \prime}\right)$ & 300.2 & 536.3 & 428.8 \\
\hline$M_{\alpha}^{j}\left(10^{9} M_{\odot}\right)$ & 1.2 & - & - \\
\hline Type & Sy 2 & LINER & LINER \\
\hline
\end{tabular}

${ }^{a}$ J2000, NED.

${ }^{b}$ Inclination angle from face-on (RC3).

${ }^{c}$ Position angle of major axis (RC3).

${ }^{d}$ Taken from Braatz et al. (1997).

${ }^{e}$ Heliocentric velocity from RC3.

${ }^{f}$ Taken from van den Bergh et al. (2002).

${ }^{g}$ AGN classification and Hubble type (NED).

${ }^{h}$ Heliocentric velocity as derived from our rotation curves.

${ }^{i}$ Distance via direct application of $v=H_{0} \cdot d\left(H_{0}=65 \mathrm{~km} \mathrm{~s}^{-1} \mathrm{Mpc}^{-1}\right)$ using velocities relative to the $3 \mathrm{~K}$ background as derived from $v_{\text {hel }}$ with the velocity calculator provided by NED.

${ }^{j}$ Estimated lower limit of mass with $\alpha=0.6$ (Lequeux 1983) within the central $2.8 \mathrm{kpc}$ (diameter).

For IC 1481, the subtraction of S0-type spectra was not satisfying as strong Balmer absorption lines and weak $\mathrm{Ca}$ II $\mathrm{H}+\mathrm{K}$ features remained. Thus, we modelled the underlying stellar continuum in two ways: i) by using the PÉGASE code (version 2.0) by Fioc \& Rocca-Volmerange (1997); and ii) by using the best fit of linear combinations of main sequence A to $F$ stars and the template spectrum of NGC 3115 . The latter provided a much better fit to the observed spectra of IC 1481, and was thus used. The fit was performed as follows:

We tried a variety of linear combinations of main sequence $\mathrm{A}$ to $\mathrm{F}$ stars on the one hand (taken from the catalogue of Pickles (1998), rebinned with a spline function to the higher resolution of our spectra), and the template spectrum of NGC 3115 on the other hand to fit the blue spectrum with its strong features and simultaneously the spectral distribution in the red range. The scaled monochromatic fluxes at $5556 \AA$ were weighted according to the different fluxes of A0V - F8V stars, and, by using a Salpeter initial mass function, normalized to the flux and mass of an A5V star.

After a first approximation of such a linear combination to gain the absorption-line free $\mathrm{H} \alpha / \mathrm{H} \beta$ value, the spectra of IC 1481 were dereddened using the recombination value for the intensity ratio $\mathrm{H} \alpha / \mathrm{H} \beta=3.1$ (a typical value for AGNs) and an average reddening curve (Osterbrock 1989, Table 7.2; MIDAS command "extin/long"). This procedure was carried out several times to find the best combination iteratively $\left(E_{B-V}=0.15\right)$ :

$$
\begin{aligned}
f_{\mathrm{IC} 1481}= & 0.099 f_{\mathrm{A} 0 \mathrm{~V}}+0.076 f_{\mathrm{A} 2 \mathrm{~V}}+0.076 f_{\mathrm{A} 3 \mathrm{~V}} \\
& +0.071 f_{\mathrm{A} 5 \mathrm{~V}}+0.064 f_{\mathrm{A} 7 \mathrm{~V}}+0.061 f_{\mathrm{F} 0 \mathrm{~V}} \\
& +0.039 f_{\mathrm{F} 5 \mathrm{~V}}+0.036 f_{\mathrm{F} 8 \mathrm{~V}}+0.48 f_{\mathrm{NGC} 3115} \\
= & 8 \times 10^{-16} \mathrm{erg} \mathrm{s}^{-1} \mathrm{~cm}^{-2} \AA^{-1} .
\end{aligned}
$$

As the lowest spectrum in Fig. 1 shows, there are no significant absorption features left that could constrain a more elaborate 

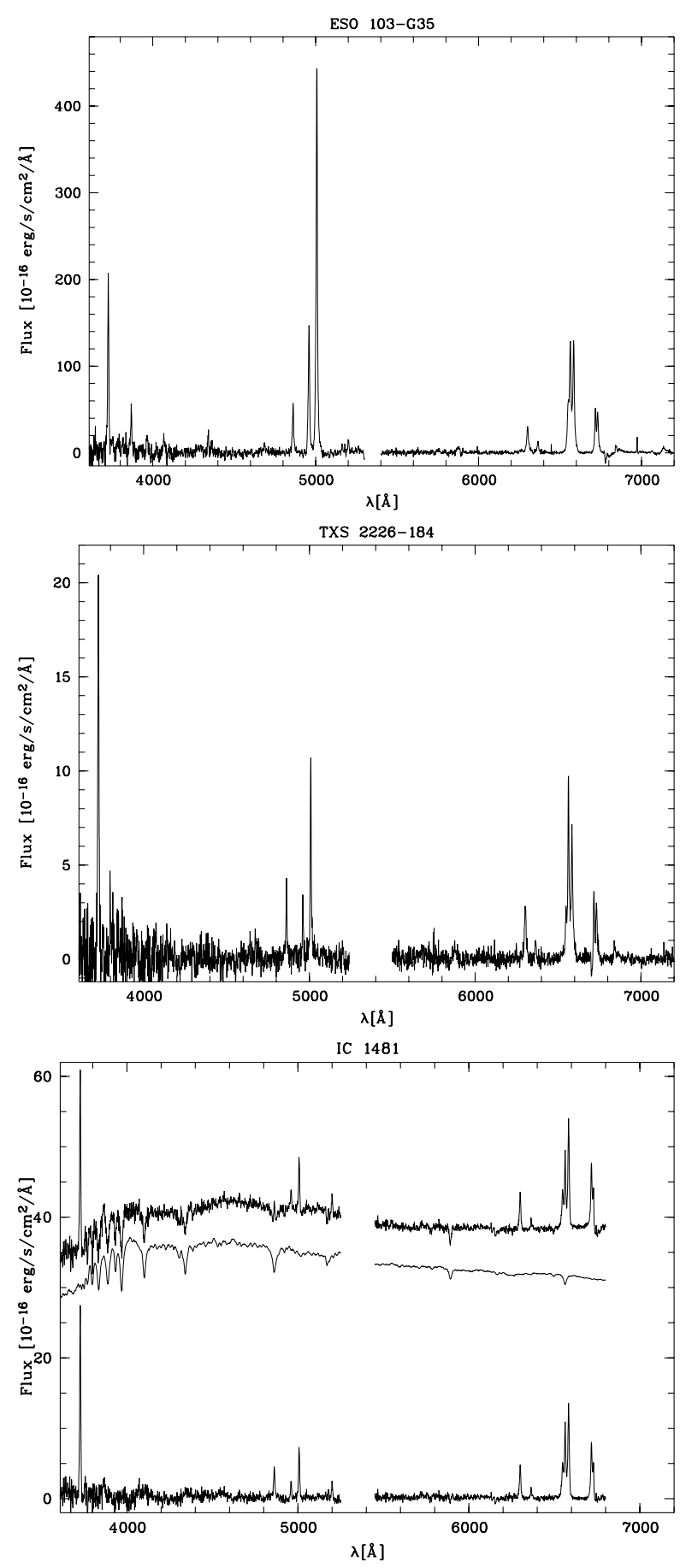

Fig. 1. Combined dereddened spectra of the red and blue wavelength range rebinned to rest wavelengths for comparison. A template galaxy spectrum (NGC 3115 or NGC 4179) was subtracted for ESO 103G035 and TXS 2226-184. The upper observed spectrum of IC 1481 shows strong Balmer absorption lines and weak $\mathrm{Ca}$ II $\mathrm{H}+\mathrm{K}$ features. Thus, a linear combination of A0V - F8V stars and the template galaxy was subtracted (see middle spectrum). The result is shown in the lower spectrum. (Both upper spectra of IC 1481 are shifted vertically by an arbitrary amount.)

population model to be subtracted. A slight depression near $\sim 4020$ A might be suggestive of He I $\lambda 4026$ from early B stars but is too noisy for detailed fitting. There is no evidence for He I $\lambda 4471$ so that we estimate a main-sequence turnoff at late
B stars. This yields a rough age of an assumed instantaneous starburst of $\sim 1.7 \times 10^{8}$ years (Iben 1967).

We converted the theoretical physical continuum fluxes of the above stars as tabulated by Cox (2000) into fluxes which would be measured at the assumed distance of $r=88.5 \mathrm{Mpc}$ (IC 1481, Table 2) using $f_{\lambda}=F_{\lambda} \frac{R^{2}}{r^{2}}\left(R=1.2-2.9 R_{\odot}\right)$ and compared them with the measured ones. This leads to $9.4 \times 10^{7}$ stars or a mass of $1.7 \times 10^{8} M_{\odot}$ in the range $\mathrm{A} 0 \mathrm{~V}$ to $\mathrm{F} 8 \mathrm{~V}$. In a more complete range for the original starburst $(\mathrm{O} 5 \mathrm{~V}-\mathrm{M} 5 \mathrm{~V}$, mass range $60-0.21 M_{\odot}$ ), this would correspond to a total mass of newborn stars of $9.9 \times 10^{8} M_{\odot}$ or $1.4 \times 10^{9}$ stars. Although these estimates are rather crude, they nevertheless show that there was a vigorous starburst in the central kiloparsec of IC 1481 $10^{8}$ years ago. This finding is contradictory to Boisson et al. (2000) who claimed that in their sample of central regions of 12 galaxies (normal galaxies, starburst galaxies, LINERs, Seyfert $1 \mathrm{~s}$ and $2 \mathrm{~s}$ ), LINERs show the oldest population with little star formation still going on. It is, however, not inconsistent with an HST image that was analyzed by Falcke et al. (2001). They speculated that the irregular shape of the galaxy might be the site of an ongoing galaxy merger.

It could be interesting to perform a detailed empirical population synthesis as proposed and developed by Bica (1988), Schmitt et al. (1996), Cid Fernandes et al. (2001), and Cid Fernandes et al. (2003) for our three galaxies and to compare the results with other stellar populations of Seyfert- 2 and LINER-host galaxies (e.g. Schmitt et al. 1999; Boisson et al. 2000; Joguet et al. 2001). However, on the one hand having a very small sample (one Sy 2 and two LINERs), we could not provide deeper insights into the links between nuclear activity and the star formation history of the host galaxy. On the other hand, as we do not expect to have missed a significant contribution of underlying stellar absorption lines, this time-consuming procedure is beyond the scope of this work.

\subsection{Rotation curves}

To gain velocity curves, we took averaged peak wavelengths of $\mathrm{H} \alpha$ and [N II] $\lambda 6583$ (with heliocentric correction applied, see Table 1) and subtracted the heliocentric systemic velocity derived by symmetrising the curve of each galaxy. This heliocentric velocity can be found in Table 2 . Rotational velocities were calculated assuming that all spectra were taken along the major axis of each galaxy. Thus, the observed velocities had to be corrected simply for the inclination angles (as taken from RC3):

$v_{\mathrm{rot}}=\frac{v_{\mathrm{obs}}}{\sin i}$.

For the central 2-4 kpc for which the $\mathrm{H} \alpha$ and [N II] $\lambda 6583$ lines were strong enough to be measured, the rotational velocities are presented in Fig. 2. The central mass could only be estimated for ESO 103-G035 (see Table 2), because the "rotation" curves for IC 1481 and TXS 2226-184 are remarkably flat. As a lower limit, we estimate the mass as

$M=\alpha \cdot \frac{v_{\mathrm{rot}}^{2} \cdot R}{G}$

with $\alpha=0.6$, taking into account non-Keplerian motion at those locations, where the gravitational potential consists of 

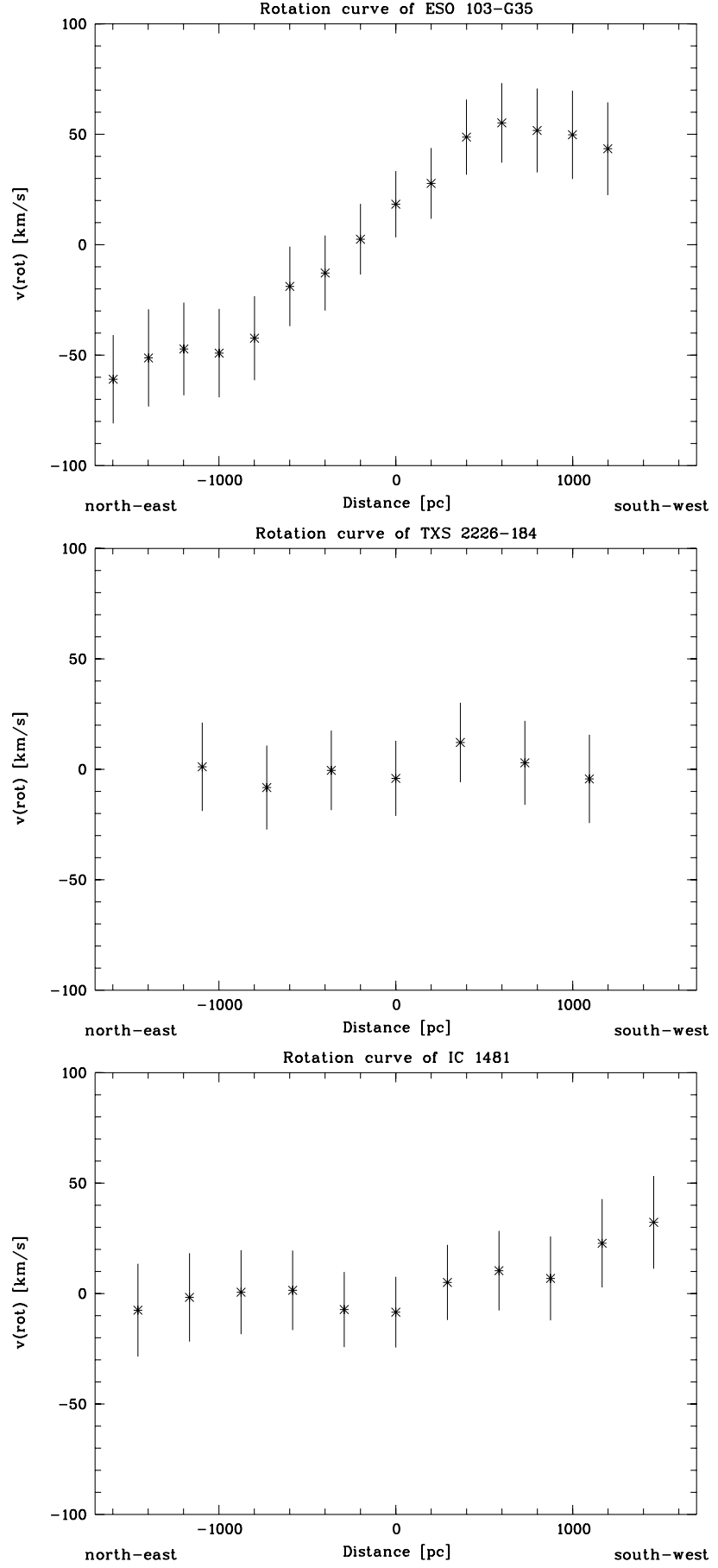

Fig. 2. Rotation curves of the three $\mathrm{H}_{2} \mathrm{O}$-megamaser galaxies derived from the average value of the $\mathrm{H} \alpha$ and [N II] $\lambda 6583$ peak wavelengths.

the superposition of a flat disk and a spherical component (Lequeux 1983). The resulting mass of ESO 103-G035 is $1.2 \times$ $10^{9} M_{\odot}$ within the central $2.8 \mathrm{kpc}$ (Table 2).

The flat "rotation" curve of IC 1481 can be explained by its amorphous appearance: The spectra were possibly not observed along the major axis, since the position of the major axis is very uncertain. The observations were done at a position angle of $\sim 24^{\circ}$ corresponding approximately to the position angle reported by Braatz et al. (1997). However, in the RC3, the position angle of the major axis is listed as $70^{\circ}$ (Table 2).
TXS 2226-184 was definetely observed along the major axis. This galaxy might therefore be an elliptical with its central gas disk lying coplanar to the major axis. While the latter is strenghtened by observations of the narrow-line region, the surface brightness distribution favors a spiral galaxy (Falcke et al. 2000). The flat rotation curve may thus be caused by the fact that our observations are confined to the extent of the nuclear bulge where gas kinematics do not follow the trend expected for a highly inclined disk that should be dominant further out.

\subsection{Emission-line profiles, intensities, and widths}

Attempts to fit the emission-line profiles of the strongest lines from $\mathrm{H},[\mathrm{OIII}],[\mathrm{OI}],[\mathrm{NII}]$, and $[\mathrm{SII}]$ in the template subtracted spectra were made with Gauss functions, Lorentz functions (=Cauchy distributions), and modified Lorentzians, dubbed here as $d$-Lorentzians, as described in Appendix A. (Note, that in the MIDAS environment, Lorentz functions are called Cauchy functions, while $d$-Lorentzians are called Lorentz functions.)

It turns out that all line profiles from the galactic nuclei had stronger wings than a single Gaussian and slightly weaker wings than a single Lorentzian, but could usually be well fit by a $d$-Lorentzian, which also allowed an appropriate decomposition of the $\mathrm{H} \alpha+[\mathrm{NII}]$ blend. In Table 3, heliocentric velocities and line widths $(F W H M)$ as well as mean $d$-parameters as derived from $d$-Lorentz fits are shown for the six strongest lines (excluding [O II] $\lambda 3727$, as it is an unresolved double line). Examples of the fits are shown in Figs. 3 and 4.

From the parameters of the $d$-Lorentz fits of strong lines, the line intensities relative to $\mathrm{H} \beta$ were derived (see Appendix A) while the intensities of faint (single) lines were directly obtained by integration using the MIDAS command "integrate/line". As for IC 1481, extinction was derived with the $c$-method (Osterbrock 1989) by using the recombination value for the intensity ratio $\mathrm{H} \alpha / \mathrm{H} \beta=3.1$ and an average reddening curve. Both observed and reddening-corrected lineintensity ratios from the central region $\left(2^{\prime \prime} \times 2^{\prime \prime}\right)$ are presented in Table 4. For pairs of lines ([O III], [O I] , and [N II]) with a fixed line ratio $(\sim 3: 1)$, only the brighter line is listed. Estimated errors of the line ratios lie in the range of $10 \%-20 \%$. Comparison with literature was only possible for the brighter lines of ESO 103-G035 (Morris \& Ward 1988), as all other line ratios have not been reported in the literature yet. Deviations of observed line ratios lie in the range of $\sim 1 \%-35 \%$ and may be due to the fact that Morris \& Ward (1988) did not use a template spectrum to subtract the underlying stellar contribution. Furthermore, they refer to a larger region $\left(1^{\prime \prime} 5 \times 5^{\prime \prime}\right)$.

Applying the classical methods outlined in Osterbrock (1989), we derived the electron density using [S II] $\lambda 6716 / \lambda 6731$ in the central region $\left(2^{\prime \prime} \times 2^{\prime \prime}\right)$. The temperature determined from [O III] $\frac{\lambda 4959+\lambda 5007}{\lambda 4363}$ only corresponds to the brightest row (formally $00^{\prime} 68 \times 2^{\prime \prime}$ ) because of the weakness of the $\lambda 4363$ line. Clearly, these parameters given in Table 5 represent some average over the central several hundred parsecs in each case (see Table 2 for the typical 
Table 3. Heliocentric velocities and line widths $(F W H M)$ (separated by $\mid$ ) in $\mathrm{km} \mathrm{s}^{-1}$ as well as mean $d$-parameter as derived from $d$-Lorentz fits for the central row.

\begin{tabular}{lccc}
\hline \hline line & ESO 103-G035 & TXS 2226-184 & IC 1481 \\
\hline $\mathrm{H} \beta$ & $4011 \mid 479$ & $7498 \mid 446$ & $6138 \mid 445$ \\
{$[\mathrm{O}$ III] $\lambda 5007$} & $4045 \mid 539$ & $7553 \mid 397$ & $6123 \mid 405$ \\
{$[\mathrm{O}$ I] $\lambda 6300$} & $4063 \mid 584$ & $7564 \mid 676$ & $6087 \mid 405$ \\
$\mathrm{H} \alpha$ & $4046 \mid 504$ & $7486 \mid 327$ & $6098 \mid 392$ \\
{$[\mathrm{~N}$ II] $\lambda 6583$} & $4046 \mid 443$ & $7486 \mid 526$ & $6097 \mid 416$ \\
{$[\mathrm{~S} \mathrm{II}] \lambda 6731$} & $4059 \mid 411$ & $7515 \mid 389$ & $6107 \mid 363^{a}$ \\
\hline mean & $4045 \pm 8 \mid 493 \pm 26$ & $7517 \pm 14 \mid 460 \pm 51$ & $6108 \pm 7 \mid 404 \pm 11$ \\
mean $d$ & $1.6 \pm 0.2$ & $1.1 \pm 0.1$ & $1.4 \pm 0.3$ \\
\hline
\end{tabular}

${ }^{a}[\mathrm{~S} \mathrm{II}] \lambda 6731$ was truncated by telluric absorption bands and thus, [S II $] \lambda 6716$ was used instead.

Table 4. Observed and reddening-corrected line intensity ratios relative to $\mathrm{H} \beta^{a}$.

\begin{tabular}{|c|c|c|c|c|c|c|}
\hline \multirow[t]{2}{*}{ Line } & \multicolumn{2}{|c|}{ ESO 103-G035 } & \multicolumn{2}{|c|}{ TXS 2226-184 } & \multicolumn{2}{|c|}{ IC 1481} \\
\hline & $F_{\text {obs }}$ & $F_{\text {dered }}$ & $F_{\text {obs }}$ & $F_{\text {dered }}$ & $F_{\text {obs }}$ & $F_{\text {dered }}$ \\
\hline [O II] $\lambda 3727$ & 1.53 & 2.56 & 4.39 & 5.69 & 3.03 & 3.43 \\
\hline$[\mathrm{Ne}$ III] $\lambda 3868$ & 0.67 & 1.03 & 0.4 & 0.49 & 0.51 & 0.57 \\
\hline $\mathrm{H} \epsilon+[\mathrm{Ne}$ III $] \lambda 3967$ & 0.22 & 0.33 & - & - & - & - \\
\hline [O III $] \lambda 4363$ & 0.12 & 0.15 & - & - & - & - \\
\hline He II $\lambda 4686$ & 0.14 & 0.15 & 0.18 & 0.19 & - & - \\
\hline [O III $] \lambda 5007$ & 9.93 & 9.44 & 2.81 & 2.74 & 1.04 & 1.02 \\
\hline$[\mathrm{Fe} \mathrm{II}]+[\mathrm{Fe}$ VII $] \lambda 5159$ & 0.13 & 0.12 & - & - & - & - \\
\hline$[\mathrm{Fe} \mathrm{VI}] \lambda 5176$ & 0.05 & 0.04 & 0.14 & 0.13 & 0.13 & 0.12 \\
\hline$[\mathrm{N}$ I $] \lambda 5199$ & 0.18 & 0.15 & 0.3 & 0.28 & 0.31 & 0.3 \\
\hline He I $\lambda 5875$ & 0.28 & 0.18 & 0.58 & 0.47 & - & - \\
\hline [O I $] \lambda 6300$ & 1.62 & 0.93 & 2 & 1.42 & 0.96 & 0.83 \\
\hline $\mathrm{H} \alpha$ & 6.11 & 3.1 & 4.45 & 3.1 & 3.66 & 3.1 \\
\hline [N II] $\lambda 6583$ & 6.4 & 3.25 & 5.13 & 3.57 & 2.36 & 2 \\
\hline [S II] $\lambda 6716$ & 2.14 & 1.05 & 1.56 & 1.05 & 1.5 & 1.26 \\
\hline [S II] $\lambda 6731$ & 2.1 & 1.03 & 1.48 & 0.98 & - & - \\
\hline Не г $\lambda 7065$ & 0.06 & 0.03 & - & - & - & - \\
\hline [Ar III] $\lambda 7136$ & 0.49 & 0.22 & - & - & - & - \\
\hline
\end{tabular}

${ }^{a}$ Derived from the central region $\left(2^{\prime \prime} \times 2^{\prime \prime}\right)$.

Table 5. Reddening-corrected $\mathrm{H} \beta$ luminosity and results from dereddened line ratios.

\begin{tabular}{lccc}
\hline \hline & ESO 103-G035 & TXS 2226-184 & IC 1481 \\
\hline$L_{\mathrm{H} \beta}{ }^{a}\left(10^{40} \mathrm{erg} \mathrm{s}^{-1}\right)$ & 2.65 & 0.49 & 0.59 \\
$A_{\mathrm{V}}{ }^{a}$ & 1.9 & 1. & 0.47 \\
$n_{\mathrm{e}}{ }^{a}\left(\mathrm{~cm}^{-3}\right)$ & $600 \pm 50$ & $550 \pm 100$ & - \\
$T^{b}(\mathrm{~K})$ & $13000 \pm 500$ & - & - \\
{$[\mathrm{O} \mathrm{III}] \lambda 5007 /[\mathrm{O} \mathrm{II}] \lambda 3727$} & 3.69 & 0.48 & 0.3 \\
{$[\mathrm{O} \mathrm{III}] \lambda 5007 /[\mathrm{O} \mathrm{I}] \lambda 6300$} & 10.15 & 2.08 & 1.23 \\
{$[\mathrm{O} \mathrm{I}] \lambda 6300 / \mathrm{H} \alpha$} & 0.3 & 0.46 & 0.27 \\
{$[\mathrm{~S} \mathrm{II}] \lambda 6716+\lambda 6731 / \mathrm{H} \alpha$} & 0.67 & 0.66 & $0.79^{c}$ \\
{$[\mathrm{~N}$ II $] \lambda 6583 / \mathrm{H} \alpha$} & 1.05 & 1.15 & 0.65 \\
\hline
\end{tabular}

${ }^{a}$ Derived from the central region $\left(2^{\prime \prime} \times 2^{\prime \prime}\right)$.

${ }^{b}$ Derived from the central row $\left(0{ }^{\prime} 68 \times 2^{\prime \prime}\right)$.

${ }^{c}$ Using an estimated ratio of [S II] $\lambda 6716 / \lambda 6731 \sim 1.07$ for a typical $n_{\mathrm{e}}$ of $\sim 550 \mathrm{~cm}^{-3}$. 

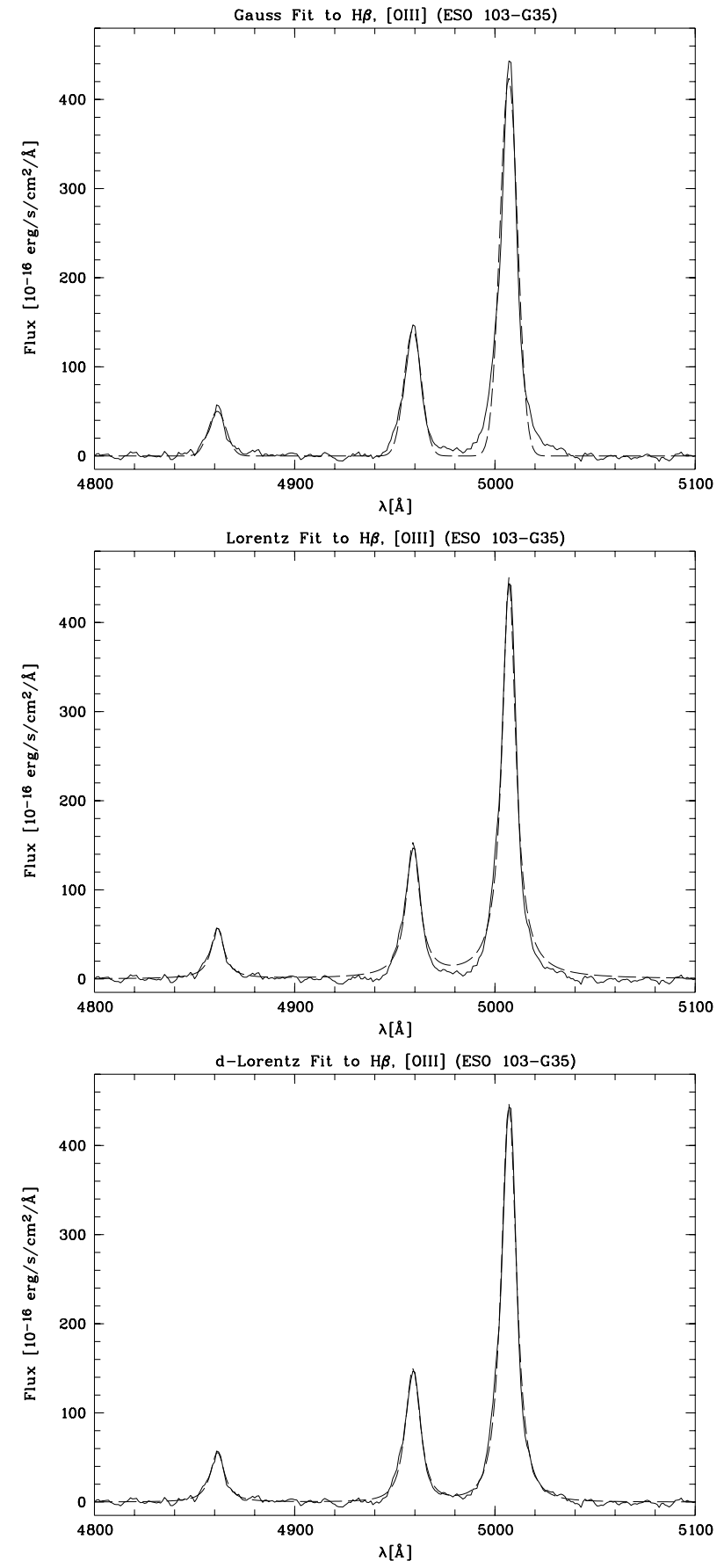

Fig. 3. $\mathrm{H} \beta$ and $[\mathrm{O} \mathrm{III}] \lambda \lambda 4959,5007$ fit by three Gaussians (upper), three Lorentzians (middle) and three $d$-Lorentzians (lower) (ESO 103G035). The total fit is shown (dashed line). Gaussians yield too narrow wings, Lorentzians too broad ones, whereas $d$-Lorentzians give the best total fit.

scale in each galaxy). For TXS 2226-184 and IC 1481, no temperature was measured, since the [O III] $\lambda 4363$ emission line was too faint. In the spectrum of IC 1481, the [S II] 16731 emission line was truncated by telluric absorption bands, thus no electron density could be derived. For TXS 2226-184, [S II] $\lambda 6716$ might be slighly affected by telluric absorption bands and the deduced electron density has to be taken with some caution. The reddening-corrected $\mathrm{H} \beta$ luminosity for the central region $\left(2^{\prime \prime} \times 2^{\prime \prime}\right)$ is given in Table 5 as well.
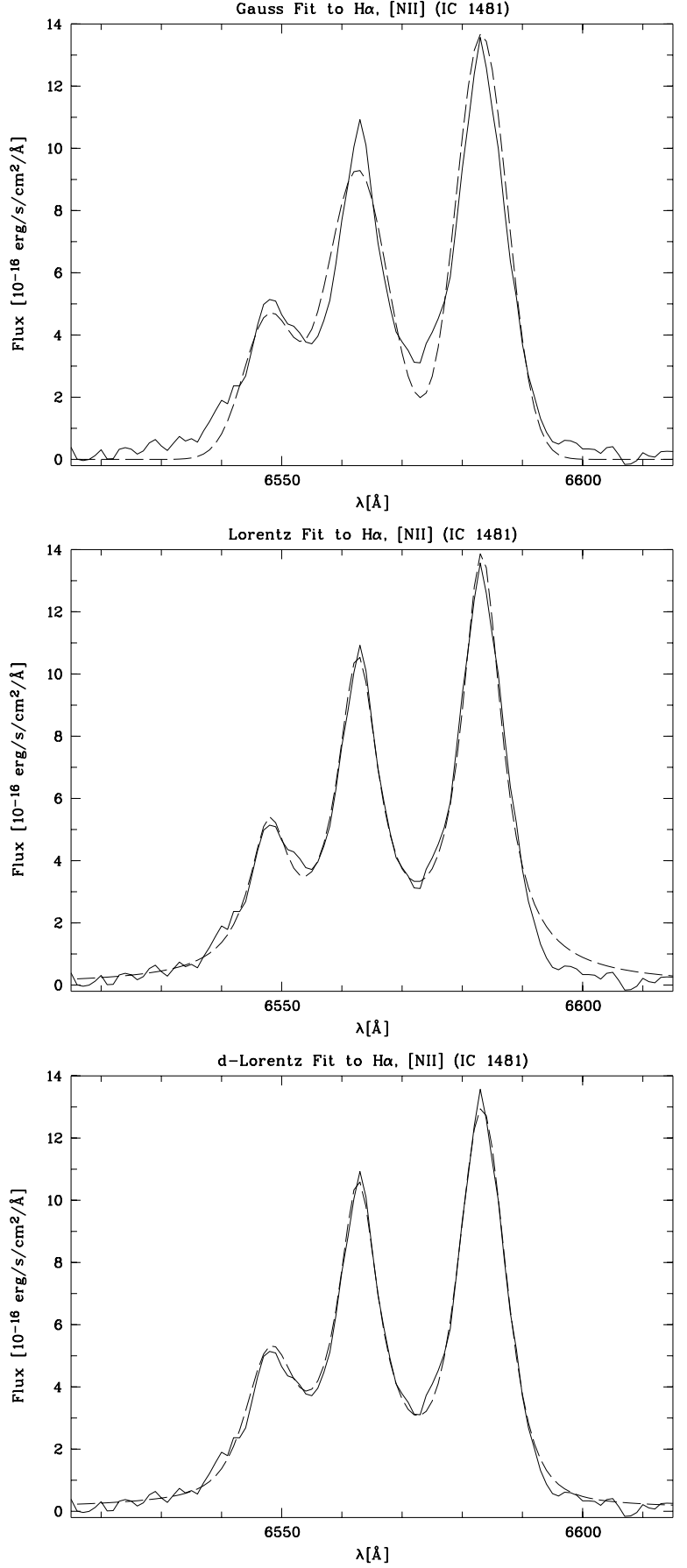

Fig. 4. The same as in Fig. 3 for the $\mathrm{H} \alpha$ and [N $\mathrm{II}] \lambda \lambda 6548$, 6583 blend (IC 1481). Again, $d$-Lorentzians lead to the best total fit.

The most important line ratios from the central region $\left(2^{\prime \prime} \times 2^{\prime \prime}\right)$ which distinguish between emission-line object classes were calculated (see Table 5) and put in the diagnostic diagrams as used in Osterbrock (1989) and Baldwin et al. (1981). ESO 103-G035 falls into the Seyfert regime. As the spectra of ESO 103-G035 did not show any broad components neither in $\mathrm{H} \alpha$ nor in $\mathrm{H} \beta$ in agreement with the results of Morris \& Ward (1988), we classify ESO 103-G035 as Seyfert-2 galaxy.

Line ratios of TXS 2226-184 and IC 1481 follow closely the LINER classification of Heckman (1980) ([O III]/[O II] $\leq 1$ 
and $[\mathrm{OIII}] /[\mathrm{OI}] \leq 3)$ and fall into the LINER regime in both the line-ratio diagrams used by Osterbrock (1989) and Baldwin et al. (1981). Furthermore, this classification is in agreement with their low luminosity, and, for IC 1481, identical with the results from Huchra et al. (1982). Our classification of TXS 2226-184 as a LINER is new.

\section{Discussion}

\subsection{Why $d$-Lorentzians?}

When discussing emission-line profiles of active galaxies, it is important to keep in mind, that they might represent lineof-sight integrations of several kinematical components such as rotating disks, cones of outflowing gas or expanding shells. Schulz et al. (1995) have shown that line-of-sight integrations through such configurations would neither yield Gaussians nor Lorentzians. Furthermore, if the spatial and spectral smearing by an instrumental function is wide compared to the kinematical gradients, the profiles will reveal a more symmetric corewing structure (Schulz et al. 1995). The latter also applies here with our spectral resolution of $\sim 130 \mathrm{~km} \mathrm{~s}^{-1}$ and spatial resolution of $\sim 1$ '. $2-2$ '. 5 which lead to an integration over relatively large cells of the phase space of an NLR.

Various observations revealed that even the "narrow" lines in AGNs can show considerable profile structure if measured at sufficient resolution (Vrtilek \& Carleton 1985; Whittle 1985; Schulz \& Henkel 2003). Commonly, in the case where singlecomponent fits fail, multi-component Gauss fits or Lorentz fits are employed.

While Gaussians are relatively well suited to describe instrumental functions, thermal Doppler broadening or some kind of turbulence, they often fail to fit broad wings. They can instead be well fit by Lorentzians, which differ from Gaussians with same $F W H M$ by more extended wings. Whittle (1985) already described the non-Gaussian nature of observed [O III] line profiles which revealed "a stronger base relative to the core than Gaussians". Véron-Cetty et al. (2001) also reported that most broad emission lines of Narrow-Line Seyfert 1 (NLS1) galaxies could be well fit by a single Lorentz profile, confirming previous claims that Lorentzians rather than Gauss profiles are better suited to reproduce the shape of the NLS1's broad emission lines. Schulz \& Henkel (2003) showed that Lorentzians appear to be better suited to fit the spectra of NGC 1052 and Mrk 1210. Hence, extended wings of intrinsic narrow-line profiles of the bulk components may not be uncommon.

However, the use of $d$-Lorentzians is new and allows us to fit both permitted and forbidden lines by adjusting the additional parameter $d$ (see Appendix A for consequences concerning the $F W H M$ ). Note that, as we are dealing here with Seyfert$2 s$ and LINERs, no broad lines were fit. In those cases in which both broad- and narrow-line components can be clearly distinguished, a multi-component fit is of course justified. According to Occam's razor, the use of $d$-Lorentzians has the advantage of adding just a single parameter instead of using multicomponent fits of Lorentzians and Gaussians. As $d$-Lorentzians seem to provide a very good fit to the data, the emission-line fluxes can be well approximated as the integral over the corresponding $d$-Lorentzian, which is a great advantage especially when measuring blended lines. Using $d$-Lorentzians does not imply a loss of physical information: on the one hand, both Lorentzians and Gaussians might not be physically motivated in all cases (as argued above) and on the other hand, the parameter $d$ gives a measure of the width of the line wings.

Our observed line wings have typically reached the noise levels at $\sim 6 \%$ of the maximum. Using Eqs. (A.1)-(A.3), a Gaussian falls to $6.3 \%$ of the maximum at 2 HWHMs (half width at half maximum) from the center, a Lorentzian to $5.9 \%$ at $4 H W H M$ s and a $d$-Lorentzian has decreased to $5.9 \%$ at 2.8 HWHM, taking a typical value of $d=1.3$. Using a mean $F W H M$ from the six strongest lines of the three galaxies (Table 3), the line wings have velocities of $600-700 \mathrm{~km} \mathrm{~s}^{-1}$. Thus, the question arises how such large velocities in the line wings can be attained. This was discussed by Schulz \& Henkel (2003) in terms of the presence of turbulence, outflows, magneto-hydrodynamic waves and electron scattering. They concluded that the latter might provide a viable explanation of the observed line wings.

For our new sample of galaxies, we may ask again, whether electron scattering can produce the observed line wing velocities, either due to the presence of hot coronal or cooler ionized gas. The ratio of scattered to input luminosity can be estimated as $L_{\mathrm{s}} / L_{\text {in }}=f \tau_{\mathrm{e}}$, with $\tau_{\mathrm{e}}=$ electron-scattering optical depth and $f=$ covering fraction of the scattering medium. To obtain $\tau_{\mathrm{e}}$ on the percent level, effective column-densities of fully ionized gas of $2 \times 10^{22} \mathrm{~cm}^{-2}$ would be required. The total bremsstrahlung luminosity of a kpc-extended sphere of a hot intercloud medium with such a column density can be estimated as $2.6 \times 10^{42}\left(n_{\mathrm{e}} / 10 \mathrm{~cm}^{-2}\right)^{2} \sqrt{T / 10^{6} \mathrm{~K}}(R / 500 \mathrm{pc})^{3} \mathrm{erg} \mathrm{s}^{-1}$ (Eq. (5.15b) in Rybicki \& Lightman 1979). The intrinsic (unabsorbed) thermal soft-X-ray emission for ESO 103-G035 (the only galaxy in our sample with measured $0.5-2 \mathrm{keV}$ flux) is estimated to be $\sim 4 \times 10^{42} \mathrm{erg} \mathrm{s}^{-1}$ (Turner et al. 1997). For a temperature of $T \sim 10^{6}$, a density of $n_{\mathrm{e}} \sim 10^{2}$ and a radius of $R \sim 1 \mathrm{kpc}$, a luminosity $\sim 500$ times higher would be expected. Thus, as in Schulz \& Henkel (2003) for NGC 1052 and Mrk 1210, we do not see enough hot coronal gas to produce the required wings in ESO 103-G035. However, electron scattering inside the cooler ionized gas component $\left(T \sim 10^{4} \mathrm{~K}\right)$ with electron densities of $n_{\mathrm{e}} \sim 500$ (which were observed for ESO 103-G035, Table 5) is a possible explanation. Calculating the expected soft-X-ray luminosity with a scale of $R \sim 10^{2} \mathrm{pc}$ yields $\sim 5 \times 10^{42} \mathrm{erg} \mathrm{s}^{-1}$, in agreement with the measurements of Turner et al. (1997). Considering a geometrical scattering factor $f \sim 0.3$, one easily obtains $L_{\mathrm{s}} / L_{\text {in }}$ of a few percent, which suffices to explain the extended wings of the narrow lines. Thus, electron scattering even at the cooler ionized gas can account for the observed velocities in the line wings.

\subsection{What triggers the water megamaser activity?}

The unique association of $\mathrm{H}_{2} \mathrm{O}$ megamasers with AGNs of Seyfert-2 and LINER type and the fact that the emission originates from the innermost parsec(s) of the parent galaxy 
(Greenhill et al. 1995a; Miyoshi et al. 1995; Claussen et al. 1998; Trotter et al. 1998; Herrnstein et al. 1999) suggest that the unknown excitation mechanism is closely related to the AGN activity (and that at least some LINERs are AGNs rather than starbursts). The Seyfert- 2 geometry is favorable for megamaser activity supplying the necessary large column densities of warm and dense molecular gas enriched with $\mathrm{H}_{2} \mathrm{O}$ molecules.

Not in all cases, however, a nuclear radio continuum background has to be amplified to obtain detectable megamaser emission (e.g. Greenhill et al. 1995a,b). Sometimes, column densities of a disk may be large enough even when viewed face-on and little is known about the solid angle of the emitted maser radiation. It is thus possible that in exceptional cases nuclear maser emission is also detectable in Seyfert-1 galaxies (Nagar et al. 2002; Hagiwara et al. 2003).

Interaction of molecular gas with radio jets will be associated with shocks and the shock heated gas favors $\mathrm{H}_{2} \mathrm{O}$ maser emission (e.g. Peck et al. 2003). Optically detectable ionized outflowing gas may, however, also trigger maser emission. Falcke et al. (2001) reported linear jet-like HST features in TXS 2226-184 and NGC 1386 that might be related to an outflow rather than an excitation cone. In the megamaser sample of Schulz \& Henkel (2003), galactic rotation and outflow of narrow-line gas are common features. The detection of $\mathrm{H}_{2} \mathrm{O}$ megamasers in very luminous infrared galaxies ( $L_{\mathrm{FIR}}>10^{11} L_{\odot}$; Hagiwara et al. 2002a; Peck et al. 2004) with presumably many young clusters of $\mathrm{O}$ and $\mathrm{B}$ stars may add further support for such an outflow scenario.

\subsubsection{Three specific cases}

There are a few nearby $\mathrm{H}_{2} \mathrm{O}$ megamaser sources that are particularly well studied, not only in view of their $\mathrm{H}_{2} \mathrm{O}$ maser properties but also at other wavelengths, including the optical and near infrared windows. Best known are the three northern of the five originally discovered megamaser sources, NGC 1068, NGC 4258 (Claussen et al. 1984), and NGC 3079 (Henkel et al. 1984; Haschick \& Baan 1985). The $\mathrm{H}_{2} \mathrm{O}$ data from NGC 4258 show a warped nuclear accretion disk that is seen almost edgeon (e.g. Miyoshi et al. 1995). Towards NGC 1068, we may view a torus with "sub-Keplerian" rotation curve (Huré 2002) that might be more massive, also with respect to the nuclear engine, and that appears to be thicker or slightly less well ordered. To the north, where the nuclear jet is bending, jet-masers are observed (e.g. Gallimore et al. 2001). NGC 3079 appears to show a disk that is less well ordered than those of NGC 1068 and NGC 4258, but a detailed map of the red-shifted maser features is still needed to obtain a complete picture (Trotter et al. 1998; Hagiwara et al. 2002b).

Direct hints for the presence of megamaser emission in these sources is given by the near infrared continuum, providing evidence for the presence of warm dust heated by the AGN. NGC 3079 harbors a compact (1") nuclear source with dust at a temperature of $\sim 1000 \mathrm{~K}$ (Israel et al. 1998). An optically detected large scale outflowing zone (Cecil et al. 2001) is apparently not triggering maser emission. An even more compact dusty core is detected in NGC 4258, with an upper size limit of 200 mas (7 pc; Chary et al. 2000). Two jets (anomalous arms) reach out of the nuclear region and shock the ambient gas in the inner $350 \mathrm{pc}$ (Wilson et al. 2001). Interestingly, the jets are much more prominent than in most other spirals, but do not trigger any megamaser emission. Instead, they provide the radio continuum background for the enhanced flux of the systemic $\mathrm{H}_{2} \mathrm{O}$ features that are stronger than the presumably self-amplified red- and blue-shifted components (Herrnstein et al. 1998). The best studied galaxy is NGC 1068 (e.g. Galliano et al. 2003). This prototypical Seyfert-2 galaxy also contains a compact near- and mid-infrared source (Marco et al. 1997; Rouan et al. 1998). The extremely red colors of its 200 mas core $(15 \mathrm{pc})$ lead to an intrinsic extinction of $A_{\mathrm{V}} \gtrsim$ $25^{\mathrm{m}}$, assuming classical grains at $1500 \mathrm{~K}$. Optical spectroscopy with the HST shows lines split into two velocity components separated by $1500 \mathrm{~km} \mathrm{~s}^{-1}$ within the inner arcsec (Axon et al. 1998). 2 micron $\mathrm{H}_{2}$ emission is also double peaked (Galliano \& Alloin 2002). Both can be explained by gas interacting with the radio jet.

To summarize, the three galaxies NGC 1068, NGC 4258, and NGC 3079 exhibit a spatially compact near infrared core containing dust clouds that are heated by the central engine. This appears to be the main hint for the potential presence of accretion disk masers, but these cores may be more difficult to detect than the masers. Tracers for jet masers are split lines at optical and near infrared wavelengths while outflow masers should show broad wings in lines arising from ionized gas as e.g. seen in NGC 1052 and Mrk 1210 (Schulz \& Henkel 2003). The nature of these outflows has, however, to be clearly identified to distinguish them from gas entrained by the much faster nuclear jets.

\subsubsection{Our data}

The new data presented here support the connection between $\mathrm{H}_{2} \mathrm{O}$ megamaser emission and the presence of Seyfert-2 and LINER nuclei. They do not, however, further elucidate the relationship between outflows and megamasers as they do not show signs of outflow in their optical spectra. In any case, establishing a connection between optical and radio data is difficult keeping in mind the different angular scales involved (i.e. a few milliarcseconds for $1.3 \mathrm{~cm}$ interferometric maps and a few arcseconds for our study), so that optical and near infrared data taken with subarcsec resolution would be highly desirable.

\subsection{3. $\mathrm{H}_{2} \mathrm{O}$ maser classification}

We conclude that all $22 \mathrm{GHz} \mathrm{H}_{2} \mathrm{O}$ masers detected so far might be subdivided into five categories with rising but often overlapping ranges of luminosity: (i) masers from latetype stars; (ii) masers from star forming regions; (iii) nuclear "outflow" masers in which outflows impinging onto dense molecular clouds may provide a suitable trigger for kilo- or megamaser emission (possibly occurring in starburst galaxies, LINERs dominated by starburst instead of AGN activity, and type-1 AGNs); (iv) "jet" masers where a direct interaction between the nuclear jet and the interstellar medium triggers maser activity; and (v) "accretion disk" masers where the masers are aligned in sometimes warped disks or thick tori. 


\section{Conclusions}

We analyzed optical spectra of the megamaser galaxies ESO 103-G035, TXS 2226-184, and IC 1481 and presented rotation curves, line ratios, electron densities, temperatures, and $L_{\mathrm{H} \beta}$. The successful line-profile decompositions of single lines as well as blends rest on $d$-Lorentzians with an additional parameter $d$ to adjust the wings, rather than Gaussians or Lorentzians as basic functions. Using $d$-Lorentzians, we can fit the extended lines of intrinsically narrow-line profiles. Crude estimates suggest that electron scattering at the ionized gas itself might lead to a viable explanation of the large line widths observed, but detailed modelling of such processes would be useful. On the basis of line ratios and their low luminosity, TXS 2226-184 and IC 1481 are classified as LINERs while ESO 103-G035 is a Seyfert-2 galaxy. No significant velocity gradient is observed along the major axis within the inner $2 \mathrm{kpc}$ of TXS 2226-184. IC 1481 reveals a post-starburst spectrum which could not be fit by an S0 template galaxy only. Instead, using additional A0V - F8V star spectra, we could correct for the Balmer absorption lines, suggestive of a vigorous starburst in the central kiloparsec $10^{8}$ years ago.

The comparison of optical spectra of all three $\mathrm{H}_{2} \mathrm{O}$ megamaser galaxies with an angular resolution of $\sim 2$ " support the connection between $\mathrm{H}_{2} \mathrm{O}$ megamaser emission and the presence of Seyfert-2 and LINER nuclei. However, the spectra do not provide direct clues to the very nuclear regions where the masers reside. Their classification as "outflow", "jet" or "accretion disk" masers remain uncertain. Apparently, either the nuclei are too heavily obscured by dust associated with cool dense foreground gas or the morphological structures giving rise to the megamaser emission, i.e. accretion disks and jets, have linear scales that are far too small to be detectable with an arcsec resolution.

Acknowledgements. In memoriam Prof. Hartmut Schulz, deceased in August 2003. N.B. remembers him gratefully for having been her "Doktorvater" in the truest sense of the word. C.H. wants to thank his friend and co-author for a fruitful and enjoyable collaboration over almost 10 years. The astronomical community has lost a highly honourable and agreeable colleague. N.B. is grateful for financial support of the "Studienstiftung des deutschen Volkes". We would like to thank the anonymous referee for useful comments and suggestions. This research has made use of the NASA/IPAC Extragalactic Database (NED), which is operated by the Jet Propulsion Laboratory, Caltech, under contract with the NASA.

\section{Appendix A: Line-profile fits}

Line profiles of the strongest lines have been fit with Gaussians, Lorentzians, and $d$-Lorentz functions as provided by MIDAS using the same initial parameters and the commands "edi/fit" and "fit/image". These basic fit functions are compared in Fig. A.1, where it is shown that Lorentzians have much stronger wings than Gaussians, but that the wings of $d$-Lorentzians can be adjusted to intermediate wing strengths. The functions are defined as ( $a=$ height, $b=$ position, $c=F W H M$
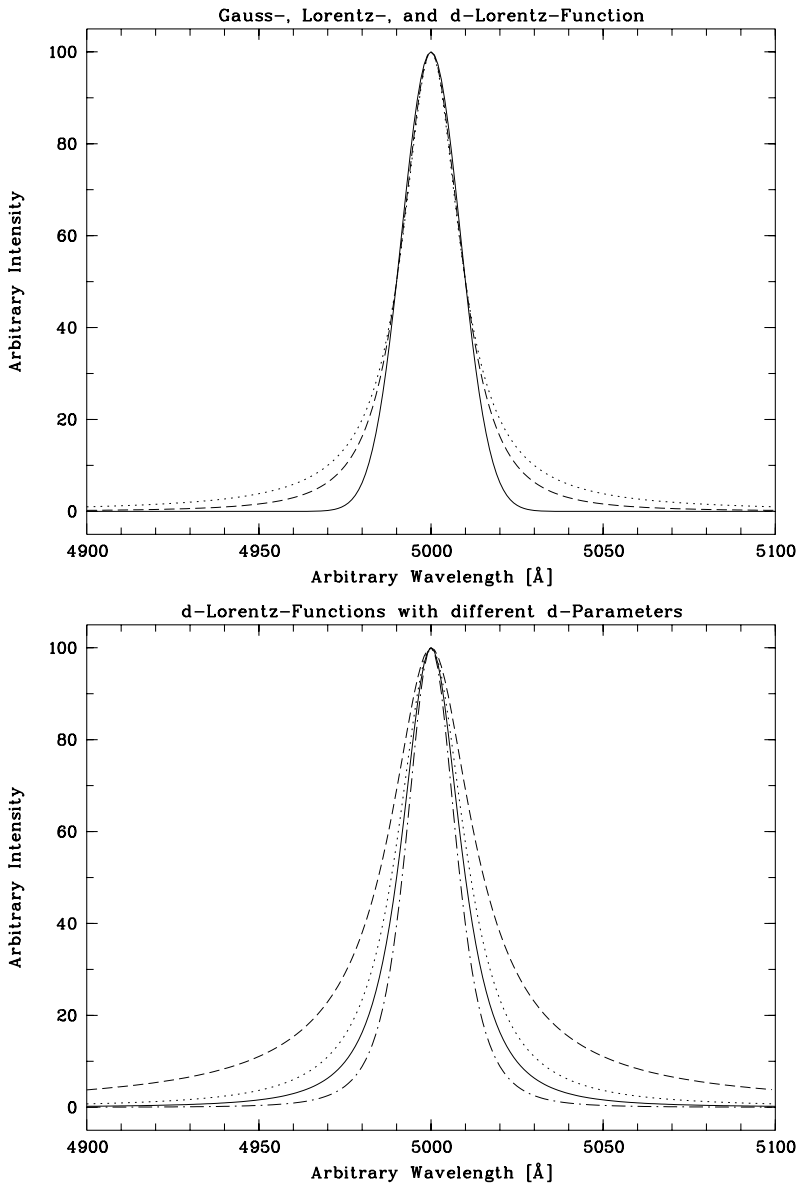

Fig. A.1. Upper panel: comparison of Gaussian (solid), Lorentzian (dotted) and $d$-Lorentz functions (dashed line) $(a=100, b=5000$, $c=20$ for Gaussian and Lorentzian, $c=26.1$ and $d=1.5$ for $d$-Lorentzian to gain the same effective $F W H M$ ). A $d$-Lorentzian falls in between a Gaussian and a Lorentz function. Lower panel: four $d$-Lorentzians are shown with different $d$ parameters $[d=0.8$ (dashed), $d=1.2$ (dotted), $d=1.5$ (solid) and $d=2$ (dash-dotted)]. With increasing $d$, the wing profile narrows. Thus, the parameter $d$ can be used to adjust the wings.

for Gaussian and Lorentzian, and $d=$ additional parameter for a $d$-Lorentzian)

Gauss $(x ; a, b, c)=a \exp \left[-\ln 2\left(\frac{2(x-b)}{c}\right)^{2}\right]$
$\operatorname{Lorentz}(x ; a, b, c)=a\left[1+\left(\frac{2(x-b)}{c}\right)^{2}\right]^{-1}$
$d$-Lorentz $(x ; a, b, c, d)=a\left[1+\left(\frac{2(x-b)}{c}\right)^{2}\right]^{-d}$.

In all galaxies, all spectral rows and for all analyzed strong emission-lines, a Gaussian yields too narrow wings, a Lorentzian too broad ones and a $d$-Lorentzian leads to the best fit (for comparison, two examples are plotted in Figs. 3 and 4). In a $d$-Lorentzian, $c$ is not the $F W H M$, which is instead given as a combination of $c$ and $d$,

$F W H M_{d \text {-Lorentz }}=c \cdot \sqrt{2^{1 / d}-1}$. 
The width at $n \%$ of the height can be calculated with

$\mathrm{W} n_{d \text {-Lorentz }}=c \cdot \sqrt{\left(\frac{1}{n}\right)^{1 / d}-1}$.

With increasing $d$ the wing-profile narrows (see Fig. A.1). The line intensities can be derived by computing the following integral

$I_{d \text {-Lorentz }}=a \cdot c \cdot \frac{\sqrt{\pi}}{2} \cdot \frac{\Gamma(-0.5+d)}{\Gamma(d)}$.

\section{References}

Antonucci, R. R. J. 1993, ARA\&A, 31, 473

Antonucci, R. R. J., \& Miller, J. S. 1985, ApJ, 297, 621

Axon, D. J., Marconi, A., Capetti, A., et al. 1998, ApJ, 496, L75

Baldwin, J. A., Phillips, M. M., \& Terlevich, R. 1981, PASP, 93, 5

Bica, E. 1988, A\&A, 195, 76

Boisson, C., Joly, M., Moultaka, J., Pelat, D., \& Serote Roos, M. 2000, A\&A, 357, 850

Bowen, I. S. 1960, ApJ, 132, 1

Braatz, J. A., Wilson, A. S., \& Henkel, C. 1996, ApJS, 106, 51

Braatz, J. A., Wilson, A. S., \& Henkel, C. 1997, ApJS, 110, 321

Cecil, G., Bland-Hawthorn, J., Veilleux, S., \& Filippenko, A. V. 2001, ApJ, 555, 338

Chary, R., Becklin, E. E., Evans, A. S., et al. 2000, ApJ, 531, 756

Cid Fernandes, R., Sodré, L., Schmitt, H. R., \& Leão, J. R. S. 2001, MNRAS, 325, 60

Cid Fernandes, R., Leão, J. R. S., \& Rodrigues Lacerda, R. 2003, MNRAS, 340, 29

Claussen, M. J., Heiligman, G. M., \& Lo, K. Y. 1984, Nature, 310, 298

Claussen, M. J., Diamond, P. J., Braatz, J. A., Wilson, A. S., \& Henkel, C. 1998, ApJ, 500, L129

Cox, A. N. (ed.) 2000, Allen's Astrophysical Quantities, Fourth Edition (New York: Springer Verlag)

De Vaucouleurs, G., De Vaucouleurs, A., Corwin, H. G., et al. 1991, Third Reference Catalogue of Bright Galaxies, III (New York: Springer Verlag) (RC3)

Falcke, H., Wilson, A. S., Henkel, C., Brunthaler, A., \& Braatz, J. A. 2000, ApJ, 530, 13

Falcke, H., Henkel, C., Wilson, A. S., \& Braatz, J. A. 2001, QSO Hosts and their Environments, ed. I. Márquez, J. Masegosa, A. Del Olmo, et al. (New York: Kluwer Academic/Plenum Publishers), 223

Fioc, M., Rocca-Volmerange, B. 1997, A\&A, 326, 950

Galliano, E., \& Alloin, D. 2002, A\&A, 393, 43

Galliano, E., Alloin, D., Granato, G. L., Villar-Martín, M. 2003, A\&A, 412,615

Gallimore, J. F., Henkel, C., Baum, S. A. et al. 2001, ApJ, 556, 694

Greenhill, L. J., Henkel, C., Becker, R., Wilson, T. L., \& Wouterloot, J. G. A. 1995a, A\&A, 304, 21

Greenhill, L. J., Jiang, D. R., Moran, J. M., et al. 1995b, ApJ, 440, 619

Greenhill, L. J., Kondratko, P. T., Lovell, J. E. J., et al. 2003, ApJ, 582, L11

Hagiwara, Y., Diamond, P. J., \& Miyoshi, M. 2002a, A\&A, 383, 65

Hagiwara, Y., Henkel, C., Sherwood, W. A., \& Baan, W. A. 2002b, A\&A, 387, L29
Hagiwara, Y., Diamond, P. J., Miyoshi, M., Rovilos, E., \& Baan, W. 2003, MNRAS, 344, L53

Haschick, A. D., \& Baan, W. A. 1985, Nature, 314, 144

Heckman, T. M. 1980, A\&A, 87, 152

Heisler, C. A., \& Vader, P. J. 1994, AJ, 107, 35

Henkel, C., Guesten, R., Downes, D., et al. 1984, A\&A, 141, L1

Herrnstein, J. R., Greenhill, L. J., Moran, J. M., et al. 1998, ApJ, 497, L69

Herrnstein, J. R., Moran, J. M., Greenhill, L. J., et al. 1999, Nature, 400,539

Huchra, J. P., Wyatt, W. F., \& Davis, M. 1982, AJ, 87, 1628

Huré, J.-M. 2002, A\&A, 395, L21

Iben, I. 1967, ARA\&A, 5, 571

Israel, F. P., van der Werf, P. P., Hawarden, T. G., \& Aspin, C. 1998, A\&A, 336, 433

Joguet, B., Kunth, D., Melnick, J., Terlevich, R., \& Terlevich, E. 2001, A\&A, 380, 19

Koekemoer, A. M., Henkel, C., Greenhill, L. J., et al. 1995, Nature, 378, 697

Lequeux, J. 1983, A\&A, 125, 394

Marco, O., Alloin, D., \& Beuzit, J. L. 1997, A\&A, 320, 399

Marshall, F. E., Boldt, E. A., Holt, S. S., et al. 1979, ApJS, 40, 657

Miyoshi, M., Moran, J. M., Herrnstein, J. R., et al. 1995, Nature, 373, 127

Morris, S. L., \& Ward, M. J. 1988, MNRAS, 230, 639

Nagar, N. M., Oliva, E., Marconi, A., \& Maiolino, R. 2002, A\&A, 391, L21

Osterbrock, D. E. O. 1989, Astrophysics of Gaseous Nebulae and Active Galactic Nuclei (University Science Books, Mill Valley)

Peck, A. B., Henkel, C., Ulvestad, J. S., et al. 2003, ApJ, 590, 149

Peck, A. B., Tarchi, A., Henkel, C., et al. 2004, in preparation

Piccinotti, G., Mushotzky, R. F., Boldt, E. A., et al. 1982, ApJ, 253, 485

Pickles, A. J. 1998, PASP, 110, 863

Rouan, D., Rigaut, F., Alloin, D., et al. 1998, A\&A, 339, 687

Rybicki, G. B., \& Lightman, A. P. 1979, Radiative Processes in Astrophysics (New York: John Wiley \& Sons)

Schmitt, H. R., Bica, E., \& Pastoriza, M. G. 1996, MNRAS, 278, 965

Schmitt, H. R., Storchi-Bergmann, T., \& Cid Fernandes, R. 1999, MNRAS, 303, 173

Schulz, H., \& Henkel, C. 2003, A\&A, 400, 41

Schulz, H., Mücke, A., Boer, B., Dresen, M., \& Schmidt-Kaler, Th. 1995, A\&AS, 109, 523

Taylor, G. B., Peck, A. B., Henkel, C., et al. 2002, ApJ, 574, 88

Trotter, A. S., Greenhill, L. J., Moran, J. M., et al. 1998, ApJ, 495, 740

Tüg, H. 1977, ESO Messenger, 11, 7

Turner, T. J., George, I. M., Nandra, K., \& Mushotzky, R. F. 1997, ApJS, 113, 23

van den Bergh, S., Weidong, L., \& Filippenko, A. V. 2002, PASP, 114, 820

Véron-Cetty, M.-P., Véron, P., \& Gonçalves, A. C. 2001, A\&A, 372, 730

Vrtilek, J. M., \& Carleton, N. P. 1985, ApJ, 294, 106

Warwick, R. S., Pounds, K. A., \& Turner, T. J. 1988, MNRAS, 231, 1145

Whittle, M. 1985, MNRAS, 213, 1

Wilson, A. S., Yang, Y., \& Cecil, G. 2001, ApJ, 560, 689 